\title{
Counterexamples to Some Results on the Existence of Field Copies ${ }^{\star}$
}

\author{
Mark A. Mostow ${ }^{1}$ and Steven Shnider ${ }^{2}$ \\ 1 Department of Mathematics, North Carolina State University, Raleigh, NC 27650, USA \\ 2 Department of Mathematics, McGill University, 805 Sherbrooke West, Montreal, P.Q., Canada \\ H3A2K 6
}

\begin{abstract}
Several criteria are known for determining which connections $A$ are determined uniquely by their curvature $F$, or by $F$ and its covariant derivatives. On a principal bundle with semi-simple gauge group $G$ over a 4-manifold $M$, a sufficient condition for $F$ to determine $A$ uniquely is that the linear map $B \rightarrow[F \wedge B]$ from Lie algebra-valued 1-forms to 3-forms (pulled back to $M$ via a local gauge) be invertible on an open dense set in $M$. Recently F. A. Doria has claimed that this condition is also necessary. We present counterexamples to this claim, and also to his assertion that $F$ determines $A$ uniquely if the restriction of the bundle to every open subset of $M$ has holonomy group.equal to $G$ and $F$ is "not degenerate as a 2-form over spacetime."
\end{abstract}

\section{Background and Results}

In recent years there has been an interest in finding criteria for the existence of field copies, that is, of gauge fields ( = field strengths = curvatures of a principal bundle) which arise from two or more gauge potentials (connections) which are not gauge equivalent (see, for example, [C, DX, DD, DW, GY, H1, H2, KC, M, R, So, W, and WY]). This problem is closely related to questions of when a connection $A$ is determined uniquely by its curvature $F$, or by $F$ together with other data (in the absolute sense, that is, ignoring gauge equivalence). Among the numerous results on these questions are two types of general criteria, which we shall call algebraic and geometric.

The algebraic criterion ([C, DT, DW, H1, MS, R]), applicable when the base space $M$ is a 4-dimensional manifold, is based on the Bianchi identity. Specialized to the trivial principal $G$-bundle $\mathbb{R}^{4} \times G$ over $M=\mathbb{R}^{4}$, it says that a sufficient condition for $F$ to determine $A$ uniquely is that the linear transformation $\operatorname{ad}_{F}: B \rightarrow[F \wedge B]$ from $\mathfrak{g}$-valued 1-forms to $\mathfrak{g}$-valued 3-forms on $M(\mathfrak{g}=$ Lie algebra of $G)$ be invertible at all $x$ in an open dense subset of $M$. (See [MS] for the generalization of this, in

^This research was supported in part by N S F grant MCS80-03419 (first author) and by an NSERCC operating grant (second author) 
bundle language, to arbitrary bundles over arbitrary 4-dimensional $M$.) The condition " $\mathrm{ad}_{F}$ is invertible on an open dense set" is a generic condition on $A$ when $G$ is semi-simple [MS], but $\mathrm{ad}_{F}$ is never invertible if the commutator subalgebra $[\mathfrak{g}, \mathfrak{g}]$ is not equal to $\mathfrak{g}$ [op. cit.].

Our first result states that the algebraic criterion, though sufficient, is not necessary. The second part of the theorem contradicts an assertion of F. A. Doria [D2, p. 2943 and p. 2949, Theorem 3(v)].

Theorem. The invertibility of $\mathrm{ad}_{F}$ on an open dense subset of $M$ is not a necessary condition for the curvature $F$ of a connection $A$ to be the curvature of no other connection $A^{\prime}$, at least when the gauge group $G$ is $\mathrm{SU}(2)$ or $\mathrm{SO}(3)$. In fact, $F$ may determine $A$ uniquely even if $\mathrm{ad}_{F}$ is nowhere invertible.

Our next result deals with geometric criteria, by which we mean the use of $F$ and of its covariant partial derivatives up to some order to determine the connection $A$. Such criteria [DD, GY, M] should involve a condition on the infinitesimal holonomy Lie algebras $\mathfrak{g}^{\prime}(p)\left[K N, \mathrm{p}\right.$. 96], where $\mathfrak{g}^{\prime}(p)$ is the linear span in $g$ at a point $p$ (in the total space $P$ of the bundle) of the components of the curvature form $F$ and of its covariant partial derivatives $D_{\mu} F, D_{\mu} D_{v} F, \ldots$ of all orders, evaluated at $p$. For example ([M, p. 141], Remark after Corollary 1$)$, if the center of $g$ is 0 (for instance, $g$ semi-simple) and if $A$ and $A^{\prime}$ are two connections for which the respective components of curvature and of covariant partial derivatives of curvature of all orders coincide and span $g$ at all $p \in P$, then $A=A^{\prime}$. This result is trivial to prove, as is the following generalization.

Proposition. Let $P \rightarrow M$ be any principal bundle $(\operatorname{dim} M$ arbitrary) with structure group $G$ whose Lie algebra $g$ has trivial center. Let $A$ and $A^{\prime}$ be two connection forms on $P$. Suppose that at all points $p \in P$, the respective components of curvature and of covariant partial derivatives of curvature of all orders less than or equal to some integer $k>1$ coincide with each other, and that for all points $p$ in an open dense subset of $P$ the components of curvature and of its covariant partial derivatives of order less than $k$ span g. Then $A=A^{\prime}$.

Proof. Adapt the proof of the quoted results [M, p. 141], and then use continuity. Q.E.D.

A result stronger than this has been asserted by F. A. Doria [D1]. He defines a holonomy condition which he calls "full irreducibility" and claims in the main result of [D1] (Theorem 3.5 and Abstract) that if it is satisfied by a connection $A$ with curvature $F$, then no other connection $A^{\prime}$ can have the same curvature, provided $F$ is not "degenerate as a 2-form over spacetime." In particular, if a connection $A$ with curvature $F$ satisfies $\mathfrak{g}^{\prime}(p)=\mathfrak{g}$ at all $p \in P$, then by standard results on holonomy ( $[\mathrm{KN}]$, or see summary in $[M, \mathrm{p} .145]$ ) the restriction of the bundle $P \rightarrow M$ to any open set $U \subset M$ has (ordinary) holonomy equal to the full group $G$ (provided that $G$ is connected). This means that $F$ is "fully irreducible." If, in addition, $F$ is nondegenerate as a 2-form on $M$, Doria's theorem would imply that $F$ determines $A$ uniquely. The following counterexample shows that the theorem cannot be true. 
Counterexample. Let $P \rightarrow M$ be the trivial bundle $\mathbb{R}^{4} \times G \rightarrow \mathbb{R}^{4}$, where $G=\mathrm{SU}(2)$ or $\mathrm{SO}(3)$. Let $\left\{t_{1}, t_{2}, t_{3}\right\}$ be a basis of $\mathrm{g}$ satisfying $\left[t_{i}, t_{j}\right]=\varepsilon_{i j k} t_{k}$. Let $x=$ $\left(x_{0}, x_{1}, x_{2}, x_{3}\right)$ be the coordinates of $\mathbb{R}^{4}$. Consider the two families of connections, parametrized by real constants $\alpha, \beta$, whose pullbacks to $\mathbb{R}^{4}$ via the trivial gauge (section) $\mathbb{R}^{4} \rightarrow \mathbb{R}^{4} \times\{1\}$ are

$$
\begin{aligned}
& A_{\alpha}=t_{1} x_{0} d x_{1}+t_{2} x_{2} d x_{3}+\alpha t_{1} d x_{3}, \\
& A_{\beta}^{\prime}=t_{1} x_{0} d x_{1}+t_{2} x_{2} d x_{3}+\beta t_{2} d x_{1} .
\end{aligned}
$$

Then all the $A_{\alpha}$ and $A_{\beta}^{\prime}$ have the curvature form

$$
F=t_{1} d x_{0} \wedge d x_{1}+t_{2} d x_{2} \wedge d x_{3}+t_{3} x_{0} x_{2} d x_{1} \wedge d x_{3}
$$

(which is non-degenerate as a 2-form on $\mathbb{R}^{4}$ ) and satisfy $\mathfrak{g}^{\prime}(p)=\mathfrak{g}$ at all points $p$. Moreover, no two of these connections are gauge equivalent to each other.

Remark 1. This Counterexample does not contradict our Proposition (taking $k=1$ ), because the first covariant partial derivatives of $F$ distinguish every pair of connections. For instance, the $A_{\alpha}$ are distinguished from each other and from $A_{\beta}^{\prime}$ by $D_{3} F_{23}=\partial_{3} F_{23}+\left[(A)_{3}, F_{23}\right]$, which equals $\alpha t_{3}$ if $A=A_{\alpha}$ and equals 0 if $A=A_{\beta}^{\prime}$.

Remark 2. For more general classes of counterexamples, see [DW], p. 391, examples 1, 2, and 3 .

\section{Proofs}

Proof of Theorem. Let $P=M \times G \rightarrow M=\mathbb{R}^{4}$ be the trivial principal $G$ bundle over $\mathbb{R}^{4}, G=\mathrm{SU}(2)$ or $\mathrm{SO}(3)$, and let $x=\left(x_{0}, x_{1}, x_{2}, x_{3}\right)$ and $\left\{t_{1}, t_{2}, t_{3}\right\}$ be as in the Counterexample above. Consider the connection (pulled back to $\mathbb{R}^{4}$ )

Its curvature is

$$
A=t_{1} d x_{0}+t_{2} d x_{1}+t_{3} x_{3} d x_{2} \text {. }
$$

$$
F=t_{3} d x_{0} \wedge d x_{1}-t_{2} x_{3} d x_{0} \wedge d x_{2}+t_{1} x_{3} d x_{1} \wedge d x_{2}-t_{3} d x_{2} \wedge d x_{3} .
$$

The linear mapping $\operatorname{ad}_{F}$ maps any $g$-valued 1 -form $B=t_{i} B_{\mu}^{i} d x_{\mu}$ (summation convention) to the $\mathfrak{g}$-valued 3 -form

$$
\begin{aligned}
{[F \wedge B]=} & L_{0} d x_{1} \wedge d x_{2} \wedge d x_{3}+L_{1} d x_{0} \wedge d x_{2} \wedge d x_{3} \\
& +L_{2} d x_{0} \wedge d x_{1} \wedge d x_{3}+L_{3} d x_{0} \wedge d x_{1} \wedge d x_{2}
\end{aligned}
$$

where the $L_{\mu}=L_{\mu}^{i} t_{i}$ are certain $g$-valued functions on $\mathbb{R}^{4}$. In particular, $L_{2}=$ $\left[t_{3}, t_{i} B_{3}^{i}\right]=t_{2} B_{3}^{1}-t_{1} B_{3}^{2}$, showing that $[F \wedge B]$ has no $t_{3} d x_{0} \wedge d x_{1} \wedge d x_{3}$ component and hence that $\operatorname{ad}_{F}$ cannot be invertible. This calculation is valid at all $x \in \mathbb{R}^{4}$.

Now suppose there were connection $A^{\prime}=A+B$ with the same curvature $F$. The two Bianchi identities $d F+[A \wedge F]=0=d F+\left[A^{\prime} \wedge F\right]$ would imply that $0=\left[F \wedge\left(A^{\prime}-A\right)\right]=[F \wedge B]$.

Lemma 1. The continuous solutions $B=B(x)$ of $[F \wedge B]=0$ are

$$
B=f_{0} t_{3} d x_{0}+f_{1} t_{3} d x_{1}+\left(f_{0} x_{3} t_{1}+f_{1} x_{3} t_{2}+f_{2} t_{3}\right) d x_{2},
$$


where $f_{0}, f_{1}, f_{2}$ are arbitrary continuous functions of $x \in \mathbb{R}^{4}$.

Proof of Lemma. The condition $[F \wedge B]=0$ is equivalent to the four equations

$$
\begin{aligned}
& 0=L_{0}=x_{3}\left[t_{1}, B_{3}\right]-\left[t_{3}, B_{1}\right], \\
& 0=L_{1}=-x_{3}\left[t_{2}, B_{3}\right]-\left[t_{3}, B_{0}\right], \\
& 0=L_{2}=\left[t_{3}, B_{3}\right], \\
& 0=L_{3}=\left[t_{3}, B_{2}\right]+x_{3}\left[t_{2}, B_{1}\right]+x_{3}\left[t_{1}, B_{0}\right],
\end{aligned}
$$

where $B_{\mu}=B_{\mu}^{i} t_{i}$. Equation (2) implies that $B_{3}^{1}=0=B_{3}^{2}$, so that

$$
B_{3}=B_{3}^{3} t_{3} \text {. }
$$

Equations (0) and (4) imply that

$$
0=L_{0}=-x_{3} B_{3}^{3} t_{2}-B_{1}^{1} t_{2}+B_{1}^{2} t_{1},
$$

and hence that

$$
0=x_{3} B_{3}^{3}+B_{1}^{1}=B_{1}^{2}
$$

Thus

$$
B_{1}=B_{1}^{i} t_{i}=-x_{3} B_{3}^{3} t_{1}+B_{1}^{3} t_{3} .
$$

Similarly, Eqs. (1) and (4) yield

$$
0=L_{1}=-x_{3} B_{3}^{3} t_{1}-B_{0}^{1} t_{2}+B_{0}^{2} t_{1},
$$

implying that

$$
B_{0}=x_{3} B_{3}^{3} t_{2}+B_{0}^{3} t_{3} .
$$

Equations (3), (7), and (9) now give

$$
0=L_{3}=B_{2}^{1} t_{2}-B_{2}^{2} t_{1}+x_{3}\left(x_{3} B_{3}^{3} t_{3}+B_{1}^{3} t_{1}\right)+x_{3}\left(x_{3} B_{3}^{3} t_{3}-B_{0}^{3} t_{2}\right) .
$$

This implies

$$
\begin{aligned}
& 0=x_{3} B_{1}^{3}-B_{2}^{2}, \\
& 0=B_{2}^{1}-x_{3} B_{0}^{3}, \\
& 0=2\left(x_{3}\right)^{2} B_{3}^{3} .
\end{aligned}
$$

Now (13) implies that $B_{3}^{3}=0$ whenever $x_{3} \neq 0$, and hence at all $x$, by continuity. This simplifies our results to

$$
B=B_{0}^{3} t_{3} d x_{0}+B_{1}^{3} t_{3} d x_{1}+\left(x_{3} B_{0}^{3} t_{1}+x_{3} B_{1}^{3} t_{2}+B_{2}^{3} t_{3}\right) d x_{2},
$$

which is equivalent to the asserted form with $f_{\mu}=B_{\mu}^{3}$ (note that $f_{3} \equiv 0$ ). Conversely, our work, read backwards, shows that any $B$ of this form satisfies $[F \wedge B]=0$.

Q.E.D. Lemma 1

Now for $A^{\prime}$ to have curvature $F, B$ must satisfy the additional condition

$$
F=d(A+B)+\frac{1}{2}[(A+B) \wedge(A+B)]
$$


or equivalently, since $F=d A+\frac{1}{2}[A \wedge A]$,

$$
0=d B+[A \wedge B]+\frac{1}{2}[B \wedge B] .
$$

As we have seen, a necessary condition for Eq. (14) to hold is $0=[F \wedge B]$. (Equivalently, one obtains $0=[F \wedge B]$ by taking $d$ of Eq. (14), just as one obtains the Bianchi identity $d F=[F \wedge A]$ by taking $d$ of the curvature equation $F=d A$ $+(1 / 2)[A \wedge A]$.

Lemma 2. For the specific connection A defined above, Eq. (14) admits only one differentiable $\left(C^{1}\right)$ solution $B$, namely $B=0$.

Proof. Since every solution $B$ of (14) satisfies $[F \wedge B]=0$, we may assume that $B$ is of the form specified in Lemma 1 , with $f_{0}, f_{1}, f_{2}$ now $C^{1}$ functions of $x \in \mathbb{R}^{4}$. Expanding Eq. (14) in components yields 18 separate equations. In particular, the coefficients of $t_{1} d x_{0} \wedge d x_{1}, t_{2} d x_{0} \wedge d x_{1}$, and $t_{1} d x_{1} \wedge d x_{2}$ are, respectively,

$$
-f_{0}=-f_{1}=x_{3}\left(\partial f_{0} / \partial x_{1}-\left(f_{1}\right)\right)^{2}+f_{2}=0,
$$

implying that $f_{0}=f_{1}=f_{2}=0$.

Q.E.D. Lemma 2 and Theorem

Proof of Counterexample. The calculation that the curvature $d A+\frac{1}{2}[A \wedge A]$ is as claimed for all $A_{\alpha}$ and $A_{\beta}^{\prime}$ is routine.

Choose one of the connections. At any point $p=(x, g) \in \mathbb{R}^{4} \times G=P, \mathfrak{g}^{\prime}(p)=$ $g^{-1} \cdot \mathfrak{g}^{\prime}(x, 1) \cdot g[\mathrm{KN}], \mathfrak{g}^{\prime}(x, 1)$ being the Lie subalgebra of $g$ spanned by the components of $F$ and its covariant partial derivatives of all orders at $x$. But the components of $F$ alone span $g$ except on $\left\{x_{0} x_{2}=0\right\}$, and even there the components of $F$ include $t_{1}$ and $t_{2}$, which generate $\mathfrak{g}$ as a Lie algebra. Hence $\mathfrak{g}^{\prime}(p)=g^{-1} \mathfrak{g} g=\mathfrak{g}$ at all $p \in P$, no matter which of the connections is used to compute the covariant partial derivatives of $F$.

The non-degeneracy of $F=F_{\mu \nu}$ as a 2-form over space-time follows from direct verification that $F_{\mu \nu} c_{\mu}=0$ only if $\left(c_{\mu}\right)=0\left(c \in \mathbb{R}^{4}\right)$.

Now if the function $\varphi: \mathbb{R}^{4} \rightarrow G$ defined a gauge transformation $\Phi$ on $P($ by $(x, g) \rightarrow$ $(x, \varphi(x) g))$, taking one of the connections to another one, then $\Phi$ would have to take $F$ to itself, that is, $\varphi^{-1} F \varphi=F$. On $\left\{x_{0} x_{2} \neq 0\right\}$ we would have $\varphi^{-1} t_{i} \varphi=t_{i}, i=1,2,3$, implying that $\varphi(x)$ is in the center of $G(1$ if $G=\operatorname{SO}(3), \pm 1$ if $G=\operatorname{SU}(2))$. But then $\Phi$ would map every connection to itself. Thus no two of the connections $A_{\alpha}, A_{\beta}^{\prime}$ can be gauge equivalent.

Q.E.D.

Acknowledgement The authors wish to thank the referee for pointing out examples in the literature which would also have served as counterexamples for our purposes

\section{References}

C Calvo, M : Connection between Yang-Mills potentials and their field strengths Phys Rev. D15 $1733-1735$ (1977)

DX Dao-xing, X : On field strengths and gauge potentials of Yang-Mills' fields. Sci Sin 20, 145-157 (1977)

DD Deser, S, Drechsler, W : Generalized gauge field copies Phys Lett 86B, 189-192 (1979) 
DT Deser, S., Teitelboim, C : Duality transforms of Abelian and non-Abelian gauge fields Phys Rev. D13, 1592-1597 (1976)

DW Deser, S., Wilczek, F : Non-uniqueness of gauge-field potentials. Phys. Lett 65B, 391-393 (1976)

D1 Doria, F A : The geometry of gauge field copies. Commun. Math. Phys 79, 435-456 (1981)

D2 Doria, F A.: Quasi-abelian and fully non-abelian gauge field copies: a classification. J Math Phys. 22, 2943-2951 (1981)

GY Gu, C -H, Yang, C -N : Some problems on the gauge field theories, II Sci Sin 20, 47-55 (1977)

H1 Halpern, M. B.: Field-strength formulation of quantum chromodynamics Phys Rev D16, 17981801 (1977)

H2 Halpern, H B.: Field strength and dual variable formulations of guage theory Phys Rev. D19, 517530 (1979)

KN Kobayashi, S, Nomizu, K.: Foundations of differential geometry, Part 1 New York: Interscience 1963

KC Kugler, M., Castillejo, L : When does the Yang-Mills field determine the potential uniquely? (Unpublished notes)

M Mostow, M A : The field copy problem: to what extent do curvature (gauge field) and its covariant derivatives determine connection (gauge potential)? Commun. Math. Phys 78, 137-150 (1980)

MS Mostow, M A, Shnider, S : Does a generic connection depend continuously on its curvature? (to appear in Commun Math Phys)

R Roskies, R : Uniqueness of Yang-Mills potentials. Phys Rev. D15, 1731-1732 (1977)

So Solomon, S : On the field strength-potential connection in non-abelian gauge theory Nucl Phys B147, 174-188 (1979)

W Weiss, $\mathbf{N}$ : Determination of Yang-Mills potentials from the field strengths Phys Rev D20, 26062609 (1979)

WY Wu, T T, Yang, C N : Some remarks about unquantized non-abelian gauge fields Phys Rev. D12, 3843-3844 (1975)

Communicated by A Jaffe

Received November 29, 1982; in revised form April 11, 1983 